\title{
Lie Symmetry Analysis, Traveling Wave Solutions, and Conservation Laws to the $(3+1)$-Dimensional Generalized B-Type Kadomtsev-Petviashvili Equation
}

\author{
Huizhang Yang $(\mathbb{D}$, Wei Liu $\mathbb{D}$, and Yunmei Zhao \\ College of Mathematics, Honghe University, Mengzi, Yunnan 661199, China \\ Correspondence should be addressed to Huizhang Yang; yanghuizhangyn@163.com
}

Received 27 July 2020; Revised 18 September 2020; Accepted 7 October 2020; Published 24 October 2020

Academic Editor: Lingzhong Guo

Copyright (c) 2020 Huizhang Yang et al. This is an open access article distributed under the Creative Commons Attribution License, which permits unrestricted use, distribution, and reproduction in any medium, provided the original work is properly cited.

\begin{abstract}
In this paper, the $(3+1)$-dimensional generalized B-type Kadomtsev-Petviashvili(BKP) equation is studied applying Lie symmetry analysis. We apply the Lie symmetry method to the $(3+1)$-dimensional generalized BKP equation and derive its symmetry reductions. Based on these symmetry reductions, some exact traveling wave solutions are obtained by using the tanh method and Kudryashov method. Finally, the conservation laws to the $(3+1)$-dimensional generalized BKP equation are presented by invoking the multiplier method.
\end{abstract}

\section{Introduction}

Nonlinear partial differential equations (NPDEs) are widely used as models to describe a great number of complex nonlinear phenomena which appear in many fields, such as hydrodynamics, biology, plasma physics, fluid dynamics, solid state physics, optics, and applied mathematics. To really understand such phenomena describing in nature, searching for exact solutions of NPDEs plays an important role in the study of nonlinear science. In recent years, more and more methods have been proposed, such as the inverse scattering method [1], the Bäcklund transform method [2], the Darboux transform method [3], the Hirota bilinear transformation method [4], the Exp-function method [5], the tanh-function method [6], the sine-Gordon equation expansion technique [7], and the Kudryashov method [8].

The Lie symmetry method presented by Lie [9] is one of the well-known methods for obtaining exact solutions of nonlinear PDEs. Up to now, the Lie symmetry method has been applied to a number of mathematical and physical models, see [10-14] and references therein. This method is effective to get similarity solutions and solitary wave solutions of NPDEs.
The study of different BKP equations has attracted a considerable size of research. Different forms of BKP equations were studied by using the Hirota method, the multiple exp-function algorithm, the Pfaffian technique, the Wronskian technique, and the Bäcklund transformation [15-21]. In this paper, by means of the Lie symmetry group method, we consider the following $(3+1)$-dimensional generalized BKP equation:

$$
u_{y t}-u_{x x x y}-3\left(u_{x} u_{y}\right)_{x}+3 u_{x x}+3 u_{z z}=0,
$$

where $u$ is a real differentiable function of the scaled spatial coordinates $x, y$, and $z$ and temporal coordinate $t$, while the subscripts denote the partial derivatives. This equation was first presented by $\mathrm{Ma}$ et al. [15]. When $z=0$, the $(3+1)$ dimensional generalized $\mathrm{BKP}$ equation can be reduced to the following $(2+1)$-dimensional BKP equation:

$$
u_{y t}-u_{x x x y}-3\left(u_{x} u_{y}\right)_{x}+3 u_{x x}=0 .
$$

Equation (1) has been investigated by different methods. Following the linear superposition principle of exponential waves, $\mathrm{Ma}$ et al. obtained an $\mathrm{N}$-wave solution for equation (1) in [15]. A bilinear Bäcklund transformation and a class of 
exact Pfaffian solutions for equation (1) were established using the Pfaffian technique and Hirotas bilinear operator identities in [16]. Moreover, Wazwaz [17] established two sets of distinct kinds of multiple-soliton solutions under specific conditions for equation (1) using the simplified form of the Hirota method. Soliton solutions in Wronskian form of (1) were also presented in [18, 19]. Multiple wave solutions and auto-Bäcklund transformation for the (1) were obtained in [20]. The lump solutions, periodic waves, and rogue waves as well as interaction solutions of (1) were obtained in [21].

As far as we know, the Lie symmetry analysis and conservation laws to the $(3+1)$-dimensional generalized BKP equation (1) have not been discussed. The main purpose of this paper is to study the Lie symmetry analysis method [22-24], exact traveling wave solutions, and conservation laws of equation (1). The rest paper is arranged as follows: Section 2 is devoted to describe the Lie symmetry vectors and symmetry reductions using Lie symmetry analysis. In Section 3, various exact traveling wave solutions are attained after reductions process by using the tanh method and Kudryashov method. Section 4 is devoted to find the conservation laws of equation (1) by utilizing the multiplier method. Some conclusions are made in Section 5.

\section{Lie Symmetry Analysis and Symmetry Reductions for Equation (1)}

We consider the one-parameter Lie group of infinitesimal transformations in $x, t, y, z$, and $u$ given by

$$
\begin{aligned}
& x \longrightarrow x+\varepsilon \xi(x, t, y, z, u), \\
& t \longrightarrow t+\varepsilon \tau(x, t, y, z, u), \\
& y \longrightarrow y+\varepsilon \eta(x, t, y, z, u), \\
& z \longrightarrow z+\varepsilon \mu(x, t, y, z, u), \\
& u \longrightarrow u+\varepsilon \phi(x, t, y, z, u),
\end{aligned}
$$

where $\epsilon$ is a group parameter. The associated vector field of equation (1) can be written as

$$
\begin{aligned}
V= & \xi(x, t, y, z, u) \frac{\partial}{\partial x}+\tau(x, t, y, z, u) \frac{\partial}{\partial t}+\eta(x, t, y, z, u) \frac{\partial}{\partial y} \\
& +\mu(x, t, y, z, u) \frac{\partial}{\partial z}+\phi(x, t, y, z, u) \frac{\partial}{\partial u}
\end{aligned}
$$

where the coefficient functions $\xi(x, t, y, z, u), \tau(x, t, y, z, u)$, $\eta(x, t, y, z, u), \mu(x, t, y, z, u)$, and $\phi(x, t, y, z, u)$ are to be determined later.

The vector field (4) is a symmetry of equation (1) if and only if

$$
\left.\operatorname{pr}^{(4)} V(\Delta)\right|_{\Delta=0}=0
$$

where $\Delta=u_{y t}-u_{x x x y}-3 u_{x x} u_{y}-3 u_{x} u_{x y}+3 u_{x x}+3 u_{z z}$ and $\mathrm{pr}^{(4)} V$ denote the fourth prolongation of $V$. Based on the Lie theory, we obtain an equivalent condition for (5) as

$$
\begin{aligned}
\operatorname{pr}^{(4)} V(\Delta)= & \phi^{y t}-\phi^{x x x y}-3 u_{y} \phi^{x x}-3 u_{x x} \phi^{y}-3 u_{x y} \phi^{x} \\
& -3 u_{x} \phi^{x y}+3 \phi^{x x}+3 \phi^{z z}=0
\end{aligned}
$$

with

$$
\begin{aligned}
\phi^{i}= & D_{i}\left(\phi-\xi u_{x}-\eta u_{y}-\mu u_{z}-\tau u_{t}\right)+\xi u_{i x}+\eta u_{i y}+\mu u_{i z} \\
& +\tau u_{i t}, \quad i=\{x, y, t y, x x, x y, z z, x x x, x x x y\}
\end{aligned}
$$

where $D_{t}, D_{x}, D_{y}$, and $D_{z}$ are total derivative operators.

Substituting (7) into (6), we can obtain the determining equations for the symmetry group of equation (1). Solving these determining equations, we conclude that the general infinitesimal symmetry of (1) has the following forms of the coefficient functions:

$$
\begin{aligned}
\xi & =-c_{1} x-3 h_{1}(t)+c_{3}, \\
\tau & =-3 c_{1} t+c_{4}, \\
\eta & =\left(c_{1}+c_{2}\right) y-c_{5} z+c_{6}, \\
\mu & =\left(-c_{1}+\frac{1}{2} c_{2}\right) z+6 c_{5} t+c_{7}, \\
\phi & =c_{1} u+h_{1}^{\prime}(t) x+c_{2} y+h_{2}(t) z+h_{3}(t),
\end{aligned}
$$

where $c_{i}(i=1, \ldots, 7)$ are arbitrary constants, $h_{1}(t), h_{2}(t)$, and $h_{3}(t)$ are arbitrary functions of $t$, and "' " is the derivative with respect to $t$. Therefore, the infinitesimal symmetries of equation (1) are

$$
\begin{aligned}
& V_{1}=\frac{\partial}{\partial x} \\
& V_{2}=\frac{\partial}{\partial t} \\
& V_{3}=\frac{\partial}{\partial y} \\
& V_{4}=\frac{\partial}{\partial z}, \\
& V_{5}=h_{2}(t) z \frac{\partial}{\partial u}, \\
& V_{6}=h_{3}(t) \frac{\partial}{\partial u}, \\
& V_{7}=-z \frac{\partial}{\partial y}+6 t \frac{\partial}{\partial z}, \\
& V_{8}=-3 h_{1}(t) \frac{\partial}{\partial x}+h_{1}^{\prime}(t) x \frac{\partial}{\partial u}, \\
& V_{9}=2 y \frac{\partial}{\partial y}+z \frac{\partial}{\partial z}+2 y \frac{\partial}{\partial u}, \\
& V_{10}=-x \frac{\partial}{\partial x}-3 t \frac{\partial}{\partial t}+y \frac{\partial}{\partial y}-z \frac{\partial}{\partial z}+u \frac{\partial}{\partial u}
\end{aligned}
$$

It can be verified that $\left\{V_{1}, \ldots, V_{9}\right\}$ is closed under the Lie bracket. The commutator table is shown in Tables 1 and 2 . 
TABle 1: Commutator table.

\begin{tabular}{lccccc}
\hline$\left[V_{i}, V_{j}\right]$ & $V_{1}$ & $V_{2}$ & $V_{3}$ & $V_{4}$ & $V_{5}$ \\
\hline$V_{1}$ & 0 & 0 & 0 & 0 & 0 \\
$V_{2}$ & 0 & 0 & 0 & 0 & $V_{5}\left(h_{2}^{\prime}(t)\right)$ \\
$V_{3}$ & 0 & 0 & 0 & 0 & 0 \\
$V_{4}$ & 0 & 0 & 0 & $-V_{6}\left(h_{2}(t)\right)$ & $V_{6}\left(h_{2}(t)\right)$ \\
$V_{5}$ & 0 & $-V_{5}\left(h_{2}^{\prime}(t)\right)$ & 0 & 0 & 0 \\
$V_{6}$ & 0 & $\left.-6 h_{3}^{\prime}(t)\right)$ & 0 & $V_{3}$ & $6 t V_{6}\left(h_{2}(t)\right)$ \\
$V_{7}$ & 0 & $-V_{8}\left(h_{1}^{\prime}(t)\right)$ & 0 & 0 & 0 \\
$V_{8}$ & $-V_{6}\left(h_{1}^{\prime}(t)\right)$ & 0 & 0 & $-V_{4}$ & $V_{5}$ \\
$V_{9}$ & 0 & $3 V_{2}$ & $-2\left[V_{3}+V_{6} / h_{3}(t)\right]$ & $-V_{4}$ & $-2 V_{5}-3 t V_{5}\left(h_{2}^{\prime}(t)\right)$ \\
$V_{10}$ & $V_{1}$ & - & $-V_{3}$
\end{tabular}

TABle 2: Commutator table.

\begin{tabular}{|c|c|c|c|c|c|}
\hline$\left[V_{i}, V_{j}\right]$ & $V_{6}$ & $V_{7}$ & $V_{8}$ & $V_{9}$ & $V_{10}$ \\
\hline$\overline{V_{1}}$ & 0 & 0 & $V_{6}\left(h_{1}^{\prime}\right)$ & 0 & $-V_{1}$ \\
\hline$V_{2}$ & $V_{6}\left(h_{3}^{\prime}(t)\right)$ & $6 V_{4}$ & $V_{8}\left(h_{1}^{\prime}\right)$ & 0 & $-3 V_{2}$ \\
\hline$V_{3}$ & 0 & 0 & 0 & $2\left[V_{3}+V_{6} / h_{3}(t)\right]$ & $V_{3}$ \\
\hline$V_{4}^{3}$ & 0 & $-V_{3}$ & 0 & $V_{4}$ & $-\hat{V}_{4}$ \\
\hline$V_{5}$ & 0 & $-6 t V_{6}\left(h_{2}(t)\right)$ & 0 & $-V_{5}^{+}$ & $2 V_{5}+3 t V_{5}^{4}\left(h_{2}^{\prime}(t)\right)$ \\
\hline$V_{6}$ & 0 & 0 & 0 & 0 & $V_{6}+3 t V_{6}\left(h_{3}^{\prime}(t)\right)$ \\
\hline$V_{7}$ & 0 & 0 & 0 & $V_{7}-2 V_{5} / h_{2}(t)$ & $2 V_{7}$ \\
\hline$V_{8}$ & 0 & 0 & 0 & 0 & $V_{8}\left(-h_{1}(t)+3 t h_{1}^{\prime}(t)\right)$ \\
\hline$V_{9}$ & 0 & $-V_{7}+2 V_{5} / h_{2}(t)$ & 0 & 0 & 0 \\
\hline$V_{10}$ & $-V_{6}-3 t V_{6}\left(h_{3}^{\prime}(t)\right)$ & $-2 V_{7}$ & $-V_{8}\left(-h_{1}(t)+3 t h_{1}^{\prime}(t)\right)$ & 0 & 0 \\
\hline
\end{tabular}

In order to derive the traveling wave solutions of (1), we consider a linear combination of the translation symmetries $V_{1}, V_{2}, V_{3}$, and $V_{4}$, namely, $V=\alpha V_{1}+V_{2}+V_{3}+V_{4}$, where $\alpha$ is a constant. Solving the corresponding characteristic equation,

$$
\frac{\mathbf{d} x}{\alpha}=\frac{\mathbf{d} y}{1}=\frac{\mathbf{d} z}{1}=\frac{\mathbf{d} t}{1}=\frac{\mathbf{d} u}{0},
$$

we obtain the following invariants:

$$
\begin{aligned}
& f=x-\alpha t, \\
& g=x-\alpha y, \\
& h=y-z, \\
& u=v(f, g, h) .
\end{aligned}
$$

Substituting (11) into (1), we can reduce the equation (1)

$$
\begin{aligned}
& \left(\alpha^{2}+6\right) v_{f g}-\alpha v_{f h}+\alpha v_{f f f g}+3 \alpha v_{f f g g}+3 \alpha v_{f g g g}+\alpha v_{g g g}-v_{f f f h}-3 v_{f f g h}-3 v_{f g g h}-v_{g g g h}+3 \alpha v_{f} v_{f g}+3 \alpha v_{f} v_{g g} \\
& +9 \alpha v_{g} v_{f g}+6 \alpha v_{g} v_{g g}+3 \alpha v_{g} v_{f f}-3 v_{f} v_{f h}-3 v_{f} v_{g h}-3 v_{g} v_{f h}-3 v_{g} v_{g h}-6 v_{h} v_{f g}-3 v_{h} v_{g g}-3 v_{h} v_{f f}+3 v_{f f}+3 v_{g g}+3 v_{h h}=0
\end{aligned}
$$

We write the vector field of (12) as

$$
\begin{aligned}
X= & \xi^{1}(f, g, h, v) \frac{\partial}{\partial f}+\xi^{2}(f, g, h, v) \frac{\partial}{\partial g}+\xi^{3}(f, g, h, v) \frac{\partial}{\partial h} \\
& +\rho(f, g, h, v) \frac{\partial}{\partial v}
\end{aligned}
$$

where the coefficient functions $\xi^{1}(f, g, h, v), \xi^{2}(f, g, h, v)$, $\xi^{3}(f, g, h, v)$, and $\rho(f, g, h, v)$ of the vector field are

$$
\begin{aligned}
\xi^{1} & =-C_{1} f+C_{2}, \\
\xi^{2} & =2 C_{1} f-3 C_{1} g+C_{3}, \\
\xi^{3} & =-3 C_{1} h+C_{4}, \\
\rho & =\left[\left(\frac{2 \alpha}{3}-\frac{4}{\alpha}\right) C_{1}-\frac{C_{5}}{\alpha}\right] f+\frac{4 C_{1}+C_{5}}{\alpha} g+C_{5} h+C_{1} v+C_{6},
\end{aligned}
$$

and $C_{i}(i=1, \ldots, 6)$ are arbitrary constants. 
We perform further symmetry reductions by applying Lie symmetries to (12), then (12) has the following six Lie symmetries:

$$
\begin{aligned}
& X_{1}=\frac{\partial}{\partial f}, \\
& X_{2}=\frac{\partial}{\partial g}, \\
& X_{3}=\frac{\partial}{\partial h}, \\
& X_{4}=\frac{\partial}{\partial v}, \\
& X_{5}=\left(-\frac{f}{\alpha}+\frac{g}{\alpha}+h\right) \frac{\partial}{\partial v}, \\
& X_{6}=-f \frac{\partial}{\partial f}+2 f \frac{\partial}{\partial g}-3 h \frac{\partial}{\partial h}+\left[\left(\frac{2 \alpha}{3}-\frac{4}{\alpha}\right) C_{1} f+\frac{4}{\alpha} g+v\right] \frac{\partial}{\partial v}
\end{aligned}
$$

We choose the symmetry $X=X_{1}+\beta X_{2}+X_{3}$, where $\beta$ is a constant. Three invariants are derived by solving the corresponding characteristic equation, namely,

$$
\begin{aligned}
r & =f-h, \\
s & =g-\beta h, \\
v(f, g, h) & =\psi(r, s) .
\end{aligned}
$$

Substituting (16) into (12), we reduce equation (12) to

$$
\begin{aligned}
& (\alpha+6) \psi_{r r}+\left(\alpha^{2}+\alpha \beta+6 \beta+6\right) \psi_{r s}+3\left(\beta^{2}+1\right) \psi_{s s}+3(\alpha+\beta+1) \psi_{r} \psi_{s s}+3(\alpha+\beta+1) \psi_{s} \psi_{r r}+6 \psi_{r} \psi_{r r} \\
& \quad+3(3 \alpha+3 \beta+1) \psi_{s} \psi_{r s}+6(\alpha+\beta) \psi_{s} \psi_{s s}+3(\alpha+\beta+3) \psi_{r} \psi_{r s}+(\alpha+\beta) \psi_{s s s s}+(3 \alpha+3 \beta+1) \psi_{r s s s}+3(\alpha+\beta+1) \psi_{r r s s} \\
& \quad+(\alpha+\beta+3) \psi_{r r r s}+\psi_{r r r r}=0 .
\end{aligned}
$$

Further symmetry reduction will transform (17) into an ODE. The symmetries of (17) include two translation symmetries $\Gamma_{1}=\partial / \partial r$ and $\Gamma_{2}=\partial / \partial s$. We consider the symmetry $\Gamma=\Gamma_{1}+\omega \Gamma_{2}$, where $\omega$ is a constant. We obtain two invariants:

$$
\begin{aligned}
\xi & =s-\omega r, \\
\psi(r, s) & =U(\xi) .
\end{aligned}
$$

Substituting (18) into (17), equation (17) is reduced to the following ODE.

$$
\mathrm{AU}^{(4)}+\mathrm{BU}^{\prime} U^{\prime \prime}+\mathrm{CU}^{\prime \prime}=0
$$

where $A=-(\omega-1)^{3}(\alpha+\beta-\omega), B=6(\omega-1)^{2}(\alpha+\beta-\omega)$, $C=(\alpha+6) \omega^{2}-\left(\alpha^{2}+\alpha \beta+6 \beta+6\right) \omega+3\left(\beta^{2}+1\right)$, and $\xi=$ $\alpha \omega t+(1-\omega) x-(\alpha+\beta-\omega) y+(\beta-\omega) z$.

\section{Traveling Wave Solutions to Equation (1)}

3.1. Exact Solutions of Equation (19) Using Tanh Method. In this subsection, we apply the tanh method to obtain solutions of equation (19). Suppose that the solution of equation (19) can be expressed as

$$
U(\xi)=\sum_{i=0}^{n} a_{i} Z^{i},
$$

where $n$ is a positive integer and $a_{i}(i=0,1,2, \ldots, n)$ are all constants. $Z=\tanh (\xi)$ is a new independent variable, the derivatives of $U$ with respect to $\xi$ can be written as

$$
\begin{aligned}
U^{\prime}= & \left(1-Z^{2}\right) \frac{\mathrm{d} U}{\mathrm{~d} Z}, \\
U^{\prime \prime}= & \left(1-Z^{2}\right)^{2} \frac{\mathrm{d}^{2} U}{\mathrm{~d} Z^{2}}-2 Z\left(1-Z^{2}\right) \frac{\mathrm{d} U}{\mathrm{~d} Z}, \\
U^{\prime \prime \prime}= & \left(1-Z^{2}\right)^{3} \frac{\mathrm{d}^{3} U}{d Z^{3}}-6 Z\left(1-Z^{2}\right)^{2} \frac{\mathrm{d}^{2} U}{\mathrm{~d} Z^{2}} \\
& +2 Z\left(1-Z^{2}\right)\left(3 Z^{2}-1\right) \frac{\mathrm{d} U}{\mathrm{~d} Z}, \\
U^{(4)}= & \left(1-Z^{2}\right)^{4} \frac{\mathrm{d}^{4} U}{d Z^{4}}-12 Z\left(1-Z^{2}\right)^{3} \frac{\mathrm{d}^{3} U}{\mathrm{~d} Z^{3}} \\
& +4\left(1-Z^{2}\right)^{2}\left(9 Z^{2}-2\right) \frac{\mathrm{d}^{2} U}{\mathrm{~d} Z^{2}}-8 Z\left(1-Z^{2}\right)\left(3 Z^{2}-2\right) \frac{\mathrm{d} U}{\mathrm{~d} Z} .
\end{aligned}
$$

Balancing the linear term of the highest order with the highest order nonlinear term, we can easily obtain $n=1$ and thus (20) becomes

$$
U=a_{0}+a_{1} Z
$$


Substituting (21) into (19) and using (22), we have an algebraic equation, which on splitting with respect to the powers of $Z$ gives the following system:

$$
\left\{\begin{array}{l}
2 a_{1}\left(-B a_{1}+12 A\right)=0, \\
-2 a_{1}\left(-2 B a_{1}+20 A-C\right)=0, \\
2 a_{1}\left(-B a_{1}+8 A-C\right)=0,
\end{array}\right.
$$

whose solution is

$$
\begin{aligned}
& a_{0} \text { is an arbitrary constant, } \\
& a_{1}=\frac{12 A}{B}=2(1-\omega), \\
& C=-4 A .
\end{aligned}
$$

Substituting (24) into (22), we obtain the traveling wave solution to equation (19)

$$
U=a_{0}+\frac{12 A}{B} \tanh (\xi) .
$$

Thus, the traveling wave solution of the $(3+1)$-dimensional generalized BKP equation (1) is

$$
\begin{aligned}
u(x, y, z, t)= & a_{0}+2(1-\omega) \tanh (\alpha \omega t+(1-\omega) x \\
& -(\alpha+\beta-\omega) y+(\beta-\omega) z),
\end{aligned}
$$

where $a_{0}, \alpha, \beta$, and $\omega$ are constants and satisfy the algebraic equation $4 A+C=4(1-\omega)^{3}(\alpha+\beta-\omega)+(\alpha+6) \omega^{2}-\left(\alpha^{2}+\right.$ $\alpha \beta+6 \beta+6) \omega+3\left(\beta^{2}+1\right)=0$.

3.2. Exact Solutions of Equation (19) Using Kudryashov Method. Suppose that the solution of equation (19) is presented as a finite series:

$$
U=\sum_{i=0}^{n} a_{i} Q^{i}(\xi),
$$

where $n$ is a positive integer and $a_{i}(i=0,1,2, \ldots, n)$ are constants, which can be determined later. Here,

$$
Q(\xi)=\frac{1}{1+\mathrm{d} a^{\xi}},
$$

which satisfies the Bernoulli equation:

$$
Q^{\prime}(\xi)=Q(\xi)(Q(\xi)-1) \ln a .
$$

Applying the homogeneous balance method similar as the tanh method, we have $n=1$. Thus, the solution (27) becomes

$$
U=a_{0}+a_{1} Q(\xi) .
$$

Substituting (30) into equation (19) together with (29), we have a system of nonlinear algebraic equations:

$$
\left\{\begin{array}{l}
2(\ln a)^{3} a_{1}\left(B a_{1}+12 A \ln a\right)=0 \\
-5(\ln a)^{3} a_{1}\left(B a_{1}+12 A \ln a\right)=0 \\
2(\ln a)^{2} a_{1}\left(25(\ln a)^{2} A+2 a_{1} B \ln a+C\right)=0 \\
-(\ln a)^{2} a_{1}\left(15(\ln a)^{2} A+a_{1} B \ln a+3 C\right)=0 \\
(\ln a)^{2} a_{1}\left((\ln a)^{2} A+C\right)=0 .
\end{array}\right.
$$

Solving this system with the help of Maple, we obtain

$$
\begin{aligned}
& a_{0}=a_{0}, \\
& a_{1}=-\frac{12 A}{B} \ln a=-2(1-\omega) \ln a, \\
& C=\frac{B}{12} \ln a .
\end{aligned}
$$

Substituting (32) into (30) together with (29), we can obtain the traveling wave solution to equation (19):

$$
U=a_{0}-\frac{12 A}{B} \frac{\ln a}{1+\mathrm{d} a^{\xi}} .
$$

Thus, the traveling wave solution of the $(3+1)$-dimensional generalized BKP equation (1) is

$$
u(x, y, z, t)=a_{0}-2(1-\omega) \frac{\ln a}{1+\mathrm{d} a^{\xi}},
$$

where $\xi=\alpha \omega t+(1-\omega) x-(\alpha+\beta-\omega) y+(\beta-\omega) z$ and $a_{0}, a, \alpha, \beta$, and $\omega$ are constants and satisfy the algebraic equation $\quad 12 C-B \ln a=12(\alpha+6) \omega^{2}-12\left(\alpha^{2}+\alpha \beta+\right.$ $6 \beta+6) \omega+36\left(\beta^{2}+1\right)-6(1-\omega)^{2}(\alpha+\beta-\omega) \ln a=0$.

\section{Conservation Laws of Equation (1)}

In this section, we study the conservation laws of the generalized $(3+1)$-dimensional BKP equation (1) using the direct multiplier method [25]. We find all the first-order multipliers $\Lambda\left(t, x, y, z, u, u_{t}, u_{x}, u_{y}, u_{z}\right)$ for the equation (1) from the determined equation:

$$
\frac{\delta}{\delta u}\left[\Lambda\left(u_{y t}-u_{x x x y}-3\left(u_{x} u_{y}\right)_{x}+3 u_{x x}+3 u_{z z}\right)\right]=0,
$$

where

$$
\begin{aligned}
\frac{\delta}{\delta u}= & \frac{\partial}{\partial u}-D_{t} \frac{\partial}{\partial u_{t}}-D_{x} \frac{\partial}{\partial u_{x}}-D_{y} \frac{\partial}{\partial u_{y}}-D_{z} \frac{\partial}{\partial u_{z}}+D_{t} D_{y} \frac{\partial}{\partial u_{t y}} \\
& +D_{x} D_{y} \frac{\partial}{\partial u_{x y}}+D_{x}^{2} \frac{\partial}{\partial u_{x x}}+D_{z}^{2} \frac{\partial}{\partial u_{z z}}+D_{x}^{3} D_{y} \frac{\partial}{\partial u_{x x x y}}
\end{aligned}
$$

denotes the Euler-Lagrange operator.

In general, for a given PDE (1), if the multiplier $\Lambda$ yields a divergence expression, 


$$
\begin{aligned}
& \Lambda\left(u_{y t}-u_{x x x y}-3\left(u_{x} u_{y}\right)_{x}+3 u_{x x}+3 u_{z z}\right) \\
& \quad=D_{t}\left(T^{t}\right)+D_{x}\left(T^{x}\right)+D_{y}\left(T^{y}\right)+D_{z}\left(T^{z}\right)
\end{aligned}
$$

for every $u$, then PDE (1) has a local conservation law in the form of $D_{t}\left(T^{t}\right)+D_{x}\left(T^{x}\right)+D_{y}\left(T^{y}\right)+D_{z}\left(T^{z}\right)=0$, where $T=\left(T^{t}, T^{x}, T^{y}, T^{z}\right)$ is a conserved vector corresponding to the conservation law.

In order to obtain the multipliers of the equation (1), expanding (35) and splitting with respect to derivative of $u$, we obtain 10 overdetermined equations as follows:

$$
\begin{aligned}
\Lambda_{u} & =0, \\
\Lambda_{u_{t}} & =0, \\
\Lambda_{u_{y}} & =0, \\
\Lambda_{u_{z}} & =0, \\
\Lambda_{x u_{x}} & =0, \\
\Lambda_{y u_{x}} & =0, \\
\Lambda_{z u_{x}} & =0, \\
\Lambda_{u u} & =0, \\
-6 \Lambda_{x}+\Lambda_{t u_{x}} & =0, \\
\Lambda_{t y}+3 \Lambda_{z z} & =0,
\end{aligned}
$$

Using the software Maple, we obtain the solution of the above overdetermined equations:

$$
\begin{aligned}
& \Lambda\left(t, x, y, z, u, u_{t}, u_{x}, u_{y}, u_{z}\right) \\
& \quad=\left(6 F_{1}(t)+C\right) u_{x}+F_{1}^{\prime}(t) x+F_{2}\left(y-t-\frac{z}{\sqrt{3}}\right),
\end{aligned}
$$

where $C$ is an arbitrary constant, $F_{1}(t)$ is an arbitrary function with respect to $t$, and $F_{2}(\xi)$ is an arbitrary function with respect to $\xi$ with $\xi=y-t-z / \sqrt{3}$. We further derive all the first-order multipliers in the following form:

$$
\begin{aligned}
\Lambda_{1} & =u_{x}, \\
\Lambda_{2} & =6 F_{1}(t)+F_{1}^{\prime}(t) x, \\
\Lambda_{3} & =F_{2}(\xi), \\
\xi & =y-t-\frac{z}{\sqrt{3}} .
\end{aligned}
$$

Corresponding to the multiplier $\Lambda_{1}=u_{x}$, we obtain the conservation law of equation (1) as

$$
D_{t}\left(T_{1}^{t}\right)+D_{x}\left(T_{1}^{x}\right)+D_{y}\left(T_{1}^{y}\right)+D_{z}\left(T_{1}^{z}\right)=0,
$$

with the following components:

$$
\begin{aligned}
T_{1}^{t}= & -\frac{1}{4} u u_{x y}+\frac{1}{4} u_{x} u_{y}, \\
T_{1}^{x}= & \frac{1}{2} u u_{t y}-\frac{1}{8} u u_{x x x y}+\frac{3}{8} u_{x x} u_{x x y}-\frac{1}{8} u_{x x x} u_{y} \\
& -\frac{5}{8} u_{x} u_{x x y}+\frac{3}{2} u_{x x}^{2}-\frac{3}{2} u_{x}^{2} u_{y}-\frac{3}{2} u_{z}^{2},
\end{aligned}
$$$$
T_{1}^{y}=-\frac{1}{4} u u_{t x}+\frac{1}{8} u u_{x x x x}-\frac{1}{2} u_{x}^{3}-\frac{1}{4} u_{x} u_{x x x}+\frac{1}{8} u_{x x}^{2}+\frac{1}{4} u_{t} u_{x},
$$$$
T_{1}^{z}=3 u_{x} u_{z}
$$

For the multiplier $\Lambda_{2}=6 F_{1}(t)+F_{1}^{\prime}(t) x$, we obtain the conservation law of equation (1) as

$$
D_{t}\left(T_{2}^{t}\right)+D_{x}\left(T_{2}^{x}\right)+D_{y}\left(T_{2}^{y}\right)+D_{z}\left(T_{2}^{z}\right)=0,
$$

with the following components:

$$
\begin{aligned}
T_{2}^{t}= & 3 F_{1}(t) u_{x} u_{y}, \\
T_{2}^{x}= & -6 F_{1}(t) u_{x} u_{x x y}-9 F_{1}(t) u_{x}^{2} u_{y}-3 F_{1}^{\prime}(t) u_{x} u_{x y} \\
& -3 F_{1}(t) u_{t} u_{y}+3 x F_{1}^{\prime}(t) u_{x}-3 F_{1}^{\prime}(t) u+9 F_{1}(t) u_{x}^{2} \\
& -9 F_{1}(t) u_{z}^{2}, \\
T_{2}^{y}= & x F_{1}^{\prime}(t) u_{t}+3 F_{1}(t) u_{x x}^{2}-x F_{1}^{\prime}(t) u_{x x x}-3 F_{1}(t) u_{x}^{3} \\
& +3 x F_{1}(t) u_{t} u_{x}, \\
T_{2}^{z}= & 18 F_{1}(t) u_{x} u_{z}+3 x F_{1}^{\prime}(t) u_{z} .
\end{aligned}
$$

For the multiplier $\Lambda_{3}=F_{2}(\xi)$, the conservation law of equation (1) is

$$
D_{t}\left(T_{3}^{t}\right)+D_{x}\left(T_{3}^{x}\right)+D_{y}\left(T_{3}^{y}\right)+D_{z}\left(T_{3}^{z}\right)=0,
$$

and the components are

$$
\begin{aligned}
T_{3}^{t} & =-\frac{1}{2} F_{2}^{\prime}(\xi) u+\frac{1}{2} F_{2}(\xi) u_{y}, \\
T_{3}^{x} & =-F_{2}(\xi) u_{x x y}-3 F_{2}(\xi) u_{x} u_{y}+3 F_{2}(\xi) u_{x}, \\
T_{3}^{y} & =\frac{1}{2} F_{2}(\xi) u_{t}+\frac{1}{2} F_{2}^{\prime}(\xi) u, \\
T_{3}^{z} & =\frac{3}{2} u_{z}+\sqrt{3} F_{2}^{\prime}(\xi) u, \\
\xi & =y-t-\frac{z}{\sqrt{3}} .
\end{aligned}
$$




\section{Conclusion}

The exact solutions of the $(3+1)$-dimensional generalized BKP equation (1) have been constructed using different methods by many authors. The Pfaffian technique, the Hirotas bilinear method, and the bilinear Bäcklund transformation were applied by many researchers, and various exact solutions such as Pfaffian solutions, lump wave solutions, periodic wave solutions, rogue wave solutions, and interaction solutions were derived. Based on the Hirota bilinear form, the Wronskian method, and the multiple Expfunction method, the Grammian and Pfaffian techniques were used to construct multiple wave solutions to the $(3+1)$ dimensional generalized BKP equation. Different from these existing results, we choose another approach to study equation (1). In this paper, the combination of Lie symmetry method and the symbolic computation is applied to equation (1). The geometric vector fields of this equation are presented for the first time in the literature. With the aid of Lie symmetry reductions, some exact traveling wave solutions are obtained by using the tanh method and Kudryashov method to the reduced equation of (1). The conservation laws of equation (1) are presented by using the multiplier method at the end of the paper. We can find that these are different approaches to find exact solutions of the $(3+1)$-dimensional generalized BKP equation, and we hope that some more interesting solutions of equation (1) are shown in the future.

\section{Data Availability}

No data were used to support this study.

\section{Conflicts of Interest}

The authors declare that there are no conflicts of interest regarding the publication of this paper.

\section{Acknowledgments}

This work was supported by the National Natural Science Foundation of China (No.11461022), Applied Basic Research Foundation of Yunnan Province (Nos. 2018FH001013 and 2018FH001-014), the Science Research Foundation of Yunnan Education Bureau (No. 2018JS479), and the Second Batch of Middle and Young Aged Academic Backbone of Honghe University (No. 2015GG0207).

\section{References}

[1] M. J. Ablowitz and P. A. Clarkson, "Solitons, nonlinear Evolution Equations and Inverse Scattering", Cambridge University Press, Cambridge, UK, 1991.

[2] C. Rogers and W. F. Shadwick, Bäcklund Transformations, Academic Press, New York, NY, USA, 1982.

[3] C. H. Gu, H. S. Hu, and Z. X. Zhou, "Darboux Transformations in Integrable Systems Theory and Their Applications to Geometry", Mathematical Physics Studies, Springer, Dordrecht, The Netherlands, 2005.
[4] R. Hirota, "Exact solution of the Korteweg-de Vries equation for multiple collisions of solitons," Physical Review Letters, vol. 27, no. 18, pp. 1192-1194, 1971.

[5] S. Zhang, "Application of exp-function method to riccati equation and new exact solutions with three arbitrary functions of Broer-Kaup-Kupershmidt equations," Physics Letters $A$, vol. 372, no. 11, pp. 1873-1880, 2008.

[6] M. A. Abdou, "The extended tanh method and its applications for solving nonlinear physical models," Applied Mathematics and Computation, vol. 190, no. 1, pp. 988-996, 2007.

[7] Y. Chen and Z. Yan, "New exact solutions of (2+1)-dimensional Gardner equation via the new sine-gordon equation expansion method," Chaos Solitons Fractals, vol. 26, no. 3, pp. 399-406, 2005.

[8] N. A. Kudryashov, "Simplest equation method to look for exact solutions of nonlinear differential equations," Chaos, Solitons \& Fractals, vol. 24, no. 5, pp. 1217-1231, 2005.

[9] S. Lie, "On integration of a class of linear partial differential equations by means of definite integrals," Archiv der Mathematik, vol. VI, no. 3, pp. 328-368, 1881.

[10] W. P. Hu, Z. Wang, Y. P. Zhao, and Z. C. Deng, "Symmetry breaking of infinite-dimensional dynamic system," Applied Mathematics Letters, vol. 103, Article ID 106207, 2020.

[11] G. W. Wang and A. M. Wazwaz, "Symmetry and painlevé analysis for the extended Sakovich equation," International Journal of Numerical Methods for Heat \& Fluid Flow, 2020.

[12] S. Kumar and D. Kumar, "Solitary wave solutions of $(3+1)$ dimensional extended Zakharov-Kuznetsov equation by lie symmetry approach," Computers \& Mathematics with Applications, vol. 77, no. 8, pp. 2096-2113, 2019.

[13] S. Kumar, D. Kumar, and A. M. Wazwaz, "Group invariant solutions of (3+1)-dimensional generalized B-type Kadomstsev Petviashvili equation using an optimal system of lie subalgebra," Physica Scripta, vol. 94, no. 6, Article ID 065204, 2019.

[14] V. Jadaun and S. Kumar, "Symmetry analysis and invariant solutions of $(3+1)$-dimensional Kadomstsev Petviashvili equation," International Journal of Geometric Methods in Modern Physics, vol. 15, no. 08, Article ID 1850125, 2018.

[15] W.-X. Ma, Y. Zhang, Y. Tang, and J. Tu, "Hirota bilinear equations with linear subspaces of solutions," Applied Mathematics and Computation, vol. 218, no. 13, pp. 71747183, 2012.

[16] M. G. Asaad and W.-X. Ma, "Pfaffian solutions to a $(3+1)-$ dimensional generalized B-type Kadomstsev Petviashvili equation and its modified counterpart," Applied Mathematics and Computation, vol. 218, no. 9, pp. 5524-5542, 2012.

[17] A. M. Wazwaz, "Distinct kinds of multiple-soliton solutions for a (3+1)-dimensional generalized B-type Kadomstsev Petviashvili equation," Physica Scripta, vol. 84, no. 5, p. 918, 2011.

[18] Y. Kang, Y. Zhang, and L. Jin, "Soliton solution to BKP equation in wronskian form," Applied Mathematics and Computation, vol. 224, no. 1, pp. 250-258, 2013.

[19] J. Su and G. J. Xu, "New exact solutions for the $(3+1)$-dimensional generalized BKP equation," Discrete Dynamics in Nature and Society, vol. 2016, p. 9, Article ID 5420156, 2016.

[20] L. Cheng and Y. Zhang, "Multiple wave solutions and autobäcklund transformation for the (3+1)-dimensional generalized B-type Kadomstsev Petviashvili equation," Computers \& Mathematics with Applications, vol. 70, no. 5, pp. 765-775, 2015.

[21] W. Li, Y. Zhang, and Y. Liu, "Exact wave solutions for a (3+1)dimensional generalized B-type Kadomstsev Petviashvili 
equation," Computers \& Mathematics with Applications, vol. 77, no. 12, pp. 3087-3101, 2019.

[22] G. Bluman and S. C. Anco, Symmetry and Integration Methods for Differential Equations, Springer, New York, NY, USA, 2002.

[23] H. Liu and J. Li, "Lie symmetry analysis and exact solutions for the short pulse equation," Nonlinear Analysis: Theory, Methods \& Applications, vol. 71, no. 5-6, pp. 2126-2133, 2009.

[24] H. Liu, J. Li, and L. Liu, "Group classifications, symmetry reductions and exact solutions to the nonlinear elastic rod equations," Advances in Applied Clifford Algebras, vol. 22, no. 1, pp. 107-122, 2012.

[25] S. C. Anco and G. Bluman, "Direct construction method for conservation laws of partial differential equations. part I: examples of conservation law classifications," European Journal of Applied Mathematics, vol. 13, no. 8, pp. 545-566, 2002. 